\title{
Determinacy of interest rate rules with bond transaction services in a cashless economy
}

\author{
Massimiliano Marzo and Paolo Zagaglia*
}

July 2008

\begin{abstract}
Canzoneri and Diba (2004) show that the Taylor principle is not a panacea for equilibrium determinacy in a model where bonds and money provide liquidity services to households. We consider a cashless variant of their model with two types of government bonds. One bond provides transaction services, whereas the other is used only as a store of value. We show that the Taylor principle is still sacrosant. In general, the results of Leeper (1991) are confirmed.
\end{abstract}

Keywords: Monetary policy, fiscal policy, government bonds, determinacy.

JEL Classification No.: E52, C68.

*Marzo: Department of Economics, Università di Bologna; massimiliano.marzo@unibo.it. Zagaglia: Research Unit, Bank of Finland, and Department of Economics, Stockholm University; Paolo.Zagaglia@bof.fi. 


\section{Introduction}

The Taylor principle has become one of the pillars of modern normative analysis of monetary policy. In a nutshell, it prescribes that the central bank should adjust the nominal rate of interest more than one-for-one as a response to changes in the inflation rate. In the standard New Keynesian model, the Taylor principle alone pins down the equilibrium inflation rate. The validity of this prescription has been challenged in the recent debate on the fiscal determinants of inflation.

The argument that the price level is determined by the degree of solvency of the government suggests that the response of monetary policy to inflation is an insufficient metric for nominal determinacy. Monetary and fiscal policy should be coordinated. In the fiscal theory of price level determination proposed by Leeper (1991), the Taylor principle should be coupled with a response of the tax rate to changes in real debt of more than one-for-one to achieve a determinate inflation rate.

One of the assumptions of Leeper (1991) is that government bonds play no direct macroeconomic role. Canzoneri and Diba (2004) and Canzoneri et al. (2006) study the interactions between monetary and fiscal policy when bonds provide transaction services for the purchase of consumption goods. This implies that there is a direct channel for government bonds to affect the inflation rate. Hence, the Taylor principle need not hold any longer for determinate equilibria to exist.

The analysis of Canzoneri and Diba (2004) and Canzoneri et al. (2006) assumes that real money balances play a key role. Not only does money enter the utility function of households. Money and bonds are imperfect substitutes. Their aggregation generates a measure of liquidity that facilitates the purchase of consumption goods.

The role of monetary aggregates in New Keynesian models is the subject of a recent debate. For instance, Woodford (2007) argues that the omission of a demand for money is not at odds with the neutrality of money. Beyond these arguments, we should stress that money demand creates an additional buffer that affects the relation the interest rate, consumption and inflation. This raises the question of the role played by money demand in the results of Canzoneri and Diba (2004).

In this paper, we follow Woodford (1998) and assume that the quantity of money that facilitates transactions is negligible. ${ }^{1}$ On the other hand, we introduce two types of bonds. One of them, which we call short-term bond, provides transaction services. The other one, labelled long-term bond, does not affect the purchase of consumption goods. We show that the results of Leeper (1991) hold also in our framework. Moreover, the Taylor principle is still sacrosant, as it requires fiscal

\footnotetext{
${ }^{1}$ This is also consistent with the presence of a 'channel system' of control of interest rates that allows an efficient management of the aggregate quantity of money (see Woodford, 2002).
} 
policy to be passive in order to pin down the price level.

This paper is organized as follows. In section 2 we present the model economy, with a focus on the portfolio allocation problem of households. In section 3, we discuss the equilibrium characteristics with explicit reference to the existence of a deflationary/inflationary equilibrium path. In section 4 , we describe the loglinearized version of the model. The calibration is presented in section 5 . The results on determinate equilibria are detailed in section 6 . Section 7 concludes.

\section{A model with bond transaction costs}

In this section we explore in a more detailed fashion the role of bonds as liquidity providers in an economy without money. In what follows, we assume that there are two categories of bonds. The first is a can be exchanged on the market at low costs, and provides the transaction services that are assigned to money in a standard monetary economy. The second type of bond is held in the households portfolio as a store of value across periods.

\subsection{Households}

There is an infinite number of agents indexed on the real line between 0 and 1 . Each $i$-th representative agent maximizes the utility function

$$
U_{t}=\sum_{t=0}^{\infty} \beta^{t} \frac{C_{i t}^{\gamma\left(1-\frac{1}{\sigma}\right)}\left(1-L_{i t}\right)^{(1-\gamma)\left(1-\frac{1}{\sigma}\right)}}{\left(1-\frac{1}{\sigma}\right)}
$$

where $C_{t}$ indicates the amount of consumption, and $L_{t}$ the amount of labor effort supplied by each single agent. In (1) $\beta$ indicates the discount factor, while $\sigma$ denotes the intertemporal substitution elasticity, the inverse of which is the coefficient of relative risk aversion.

We assume the existence of a large number of differentiated goods indexed over the real line between 0 and 1 . This allows each firm to have a control of the price of her final good to be sold, since output becomes demand determined. Following the approach by Dixit and Stiglitz (1977), we assume that the consumption bundle $C_{i t}$ demanded by each agent $i \in[0,1]$ is a CES type aggregate of all the $j \in[0,1]$ varieties of final goods produced in this economy

$$
C_{i t}=\left[\int_{0}^{1} c_{t}^{i}(j)^{\frac{\theta-1}{\theta}} d j\right]^{\frac{\theta}{\theta-1}}
$$

where $\theta$ is the elasticity of substitution between different varieties of goods produced 
by each firm $j$. To guarantee the existence of an equilibrium, the elasticity $\theta$ is restricted to be bigger than one. Standard optimization problem for the choice of the optimal composition of bundle (2) lead to the constant-elasticity inverse demand function

$$
\frac{c_{t}^{i}(j)}{C_{i t}}=\left[\frac{p_{t}(j)}{P_{t}}\right]^{-\theta}
$$

where $p_{t}(j)$ is the price of variety $j$ and $P_{t}$ is the general price index defined as

$$
P_{t}=\left[\int_{0}^{1} p_{t}(j)^{1-\theta} d j\right]^{\frac{1}{1-\theta}}
$$

The aggregate price level $P_{t}$ is beyond the control of each individual firm.

When we aggregate $C_{i t}$ and $c_{t}^{i}(j)$ across all agents $i$, we obtain the aggregate demand for final goods and for variety $j$ given by $Y_{t}=\int_{0}^{1} C_{i t} d i$ and $Y_{j t}=\int_{0}^{1} c_{t}^{i}(j) d i$, for all $j \in[0,1]$.

\subsection{Portfolio choice}

The budget constraint is

$$
\begin{aligned}
B_{i t}+B_{i t}^{*}=\left(1+i_{t-1}^{*}\right) B_{i t-1}^{*} & +\left(1+i_{t-1}\right) B_{i t-1} \\
+ & W_{i t} L_{i t}+P_{t} \Omega_{i t}^{j}-P_{t} C_{i t}\left(1+\chi g\left(F_{i t}\right)\right)-T_{i t}
\end{aligned}
$$

The households allocates resources between two types of bonds, $B_{i t}^{*}$ and $B_{i t}$. Bonds $B_{i t}^{*}$ are standard in that they do not provide any type of transaction service. This security pays an interest rate $i_{t-1}^{*}$. Bonds $B_{i t}$, can be used to purchase consumption goods through the transaction technology $f\left(F_{i t}\right)$. The term $F_{i t}$ is the 'velocity of circulation of bonds', and is defined as

$$
F_{i t}=\frac{P_{t} C_{i t}}{B_{i t}}
$$

From (6) we observe that $F_{i t}$ plays the same role of the velocity of circulation of money. In particular, the function $g\left(F_{i t}\right)$ is required to have the properties

$$
\begin{aligned}
g\left(F_{i t}\right) & =0 \text { for } F_{i t} \leq 0 \\
g^{\prime}\left(F_{i t}\right) & >0 \text { and } g^{\prime \prime}\left(F_{i t}\right) \geq 0
\end{aligned}
$$

Assumption (7) tells us that negative bond holdings do not provide any transaction services. Assumption (8), instead, shows that the transaction cost function is in- 
creasing and convex in $F_{i t}$. The convexity of $g\left(F_{i t}\right)$ is needed to make sure that the utility maximization program delivers a true maximum.

Summing up, the term $\chi g\left(F_{i t}\right)$ introduces transaction costs in terms of consumption spending, with a constant scale parameter $\chi$. Implicit in our formulation there is the assumption that the economy under analysis possesses an almost perfect mechanism to shift funds from one checking account to another by changing the portfolio composition of government bonds holdings.

Finally, from equation (5) we have that in addition to income derived from investment in bonds $\left(i_{t-1} B_{t-1}\right.$ and $\left.i_{t-1}^{*} B_{t-1}^{*}\right)$, each agent derives funds from supplying labor in quantity $L_{i t}$, paid at the wage rate $W_{i t}$. An additional source of income is the participation to the profit of $j$-th firm producing the final good variety $j, \Omega_{i t}^{j}$. A lump sum tax denoted by $T_{i t}$ is levied on the income of the household.

\subsection{Optimality conditions for households}

The first order conditions with respect to $C_{i t}, L_{i t}, B_{i t}, B_{i t}^{*}$, respectively

$$
\begin{aligned}
\gamma C_{i t}^{\gamma\left(1-\frac{1}{\sigma}\right)-1}\left(1-L_{i t}\right)^{(1-\gamma)\left(1-\frac{1}{\sigma}\right)} & =P_{t} \lambda_{i t}\left[1+\chi g\left(F_{i t}\right)+\chi g^{\prime}\left(F_{i t}\right) F_{i \in}(9)\right. \\
(1-\gamma) C_{i t}^{\gamma\left(1-\frac{1}{\sigma}\right)}\left(1-L_{i t}\right)^{(1-\gamma)\left(1-\frac{1}{\sigma}\right)-1} & =\lambda_{i t} W_{i t} \\
\beta E_{t} \frac{\lambda_{i t+1}}{P_{t+1}}\left(1+i_{t}^{*}\right) & =\frac{\lambda_{i t}}{P_{t}} \\
\beta E_{t} \frac{\lambda_{i t+1}}{P_{t+1}}\left(1+i_{t}\right) & =\frac{\lambda_{i t}}{P_{t}}\left[1-\chi g^{\prime}\left(F_{i t}\right) F_{i t}^{2}\right] \\
\lambda_{i t} B_{i t} & =0, \quad \lambda_{i t} \geq 0 \\
\lambda_{i t} B_{i t}^{*} & =0, \quad \lambda_{i t} \geq 0
\end{aligned}
$$

In (9)-(14) $\lambda_{i t}$ indicates the Lagrange multiplier associated to constraint (5). Equation (9) defines the intertemporal choice of consumption, where the effects of first order due to the transaction technology appear a critical element in the definition of intertemporal trade-offs. Equation (10) defines the optimal labor supply choice and equates the disutility from work effort to the real wage weighted by the marginal utility of consumption. Equation (11) indicates the optimal allocation of bonds $B_{i t-1}^{*}$, while equation (12) represents the optimal allocation of bonds $B_{i t}$. In particular, from equation (12) we observe that the presence of transaction costs generates a wedge between left and right hand side. 


\subsection{A disgression on the transaction cost function for bonds}

In what follows, we assume that the functional form for the transaction cost function $g\left(F_{i t}\right)$ is

$$
g\left(F_{i t}\right)=\frac{F_{i t}^{2}}{2}
$$

Function (15) fulfills all the requirements stated in (7) and (8) and allows a tractable derivation of the equilibrium conditions. Therefore, using (15) into (9) and (12) and rearranging terms, we can derive the demand for short term bonds

$$
b_{i t}=\left[\chi \frac{\left(1+i_{t}\right)}{i_{t}^{*}-i_{t}}\right]^{1 / 3} C_{i t}
$$

where $b_{i t}=B_{i t} / P_{t}$. It is immediate to verify that short term bond demand (16) is (i) increasing with respect to $i_{t}$ and $C_{i t}$, and (ii) decreasing with respect to $i_{t}^{*}$. In fact, if the return on short-term bonds rises, they become more attractive with respect to long-term bonds. If consumption increases, then the demand for short term bonds increases, because of the need to finance a wider number of transactions. Finally, if the return on long-term bonds $i_{t}^{*}$ increases, the demand for $B_{i t}$ reduces. Equation (16) is similar to a traditional money demand function with money instead of short-term bonds. It should be pointed out that equation (16) is defined only for a positive spread between the rates.

The imperfect substitutability existing between the two types of bonds is already reflected into the transaction role attached to the quantities of short-term bonds. However, in order to obtain simple closed-form solutions, we assume that the rate of returns on long-term bonds follows a close relationship with the rate of return on short-term bonds. In particular, we assume that the relationship existing between the rate of returns of the two types of bonds is

$$
i_{t}^{*}=\zeta_{t} i_{t}
$$

where $\zeta_{t}$ is a stochastic term representing the comovements existing between the long rate $i_{t}^{*}$ and the short term rate $i_{t}$. The term $\zeta_{t}$ can be represented according to the stochastic process

$$
\log \zeta_{t}=\left(1-\rho_{\zeta}\right) \zeta+\rho_{\zeta} \log \zeta_{t-1}+\varepsilon_{\zeta t}
$$

where $\zeta$ is the steady state level of $\zeta_{t}$ and $\varepsilon_{\zeta t}$ is a random variable i.i.d. and normally distributed as $N\left(0, \sigma_{\zeta}^{2}\right)$, and the autoregressive coefficient $\rho_{\zeta}$ is assumed to be less than one. 


\subsection{The production side}

Staggered pricing arises from menu costs along the lines of Rotemberg (1982). Under this approach, prices changes are costly according to

$$
A C_{t}^{p}=\frac{\phi_{p}}{2}\left[\frac{P_{t}(j)}{P_{t-1}(j)}-\pi\right]^{2} Y_{t}
$$

where $\pi$ is the steady state of the inflation rate and $\phi_{p}$ is the parameter of the adjustment cost function $A C_{t}^{p}$ that determines the level of price rigidity existing in the model. Under (19) a firm pays a cost in terms of output $Y_{t}$ each time the price level of her final goods differs from steady state inflation rate $\pi$.

The production function of each variety $j$ is

$$
Y_{t}(j)=A_{t} L_{t}^{\alpha}(j)
$$

According to (20) each firm employs a given quantity of labor $L_{t}(j)$ in the production of $j$-variety. Note that all firms producing $j$ varieties are subject to a technology shock $A_{t}$

$$
\log A_{t}=\left(1-\rho_{A}\right) A+\rho_{A} \log A_{t-1}+\varepsilon_{A t}
$$

Where $\rho_{A}<1$ and $\varepsilon_{A t}$ is an i.i.d. random variable, normally distributed with zero mean and variance $\sigma_{a}^{2}$.

Each individual firm faces a downward demand curve of the same demand (3), with $Y_{t}(j)$ in place of $C_{i t}$ and chooses the optimal quantity of labor input $L_{t}(j)$ by maximizing the stream of real profits

$$
\Omega_{t}(j)=\sum_{t=0}^{\infty} \rho_{t} \omega_{t}(j)=\sum_{t=0}^{\infty} \rho_{t}\left[\frac{P_{t}(j)}{P_{t}} Y_{t}(j)-\frac{W_{t}}{P_{t}} L_{t}(j)-A C_{t}^{p}\right]
$$

subject to the demand function (3), to the production function (20) and to the price adjustment cost function (19). Note that in (22) $\rho_{t}$ is a stochastic pricing kernel for contingent claims employed by firms to discount future profit stream. Thus, the firm's first order condition with respect to $L_{t}(j)$ is

$$
\frac{W_{t}}{P_{t}}=\alpha\left(1-\frac{1}{\psi_{t}(j)}\right)\left(\frac{Y_{t}(j)}{L_{t}(j)}\right)\left(\frac{P_{t}(j)}{P_{t}}\right)
$$


where

$$
\begin{aligned}
\frac{1}{\psi_{t}(j)}= & \frac{1}{\theta}\left\{1-\phi_{p}\left[\frac{P_{t}(j)}{P_{t-1}(j)}-\pi\right] \frac{P_{t}}{P_{t-1}(j)} \frac{Y_{t}}{Y_{t}(j)}\right. \\
& \left.+E_{t}\left[\phi_{p} \frac{\rho_{t+1}}{\rho_{t}}\left(\frac{P_{t+1}(j)}{P_{t}(j)}-\pi\right) \frac{P_{t+1}}{P_{t}(j)} \frac{P_{t+1}(j)}{P_{t}(j)} \frac{Y_{t+1}}{Y_{t}(j)}\right]\right\}
\end{aligned}
$$

In (47)- $(24), \psi_{t}(j)$ can be interpreted as the output demand elasticity augmented by cost of price adjustment weighted by the stochastic pricing kernel $\rho_{t}$. In steady

state, if $\frac{P_{t}(j)}{P_{t-1}(j)}=\pi$ for all $t$ and $j$, we get that $\psi_{t}(j)=\theta$. The markup for each firm $j$ is defined by

$$
\mu_{t}(j)=\left(1-\frac{1}{\psi_{t}(j)}\right)^{-1}
$$

In steady state, the markup is $\frac{\theta}{\theta-1}$, so that when $\theta \rightarrow \infty, \mu \rightarrow 1$. With perfectly flexible prices with $\phi_{p}=0$ the markup is once again $\frac{\theta}{\theta-1}$.

\subsection{Fiscal policy}

The government budget constraint can be written as

$$
B_{t}+B_{t}^{*}=\left(1+i_{t-1}\right) B_{t-1}+\left(1+i_{t-1}^{*}\right) B_{t-1}^{*}+G_{t}-T_{t}
$$

In (26) the primary deficit (surplus) $G_{t}-T_{t}$ plus interest rate proceedings paid by the government to the owner of government debt (both short and long debt) $i_{t-1} B_{t-1}$, $i_{t-1}^{*} B_{t-1}^{*}$, are financed by issuing new debt, namely $B_{t}-B_{t-1}, B_{t}^{*}-B_{t-1}^{*}$.

The equilibrium condition for government bonds suggests that the demand equals supply at each point in time. Therefore, we can write

$$
\begin{aligned}
B_{t} & =\int_{0}^{1} B_{i t} d i \\
B_{t}^{*} & =\int_{0}^{1} B_{i t}^{*} d i \\
T_{t} & =\int_{0}^{1} T_{i t} d i
\end{aligned}
$$

We assume the existence of a fiscal policy rule that sets the level of taxes in reaction to the outstanding level of real debt

$$
T_{t}=\psi_{0}+\psi_{1} \frac{B_{t-1}^{*}}{P_{t}}+\psi_{2} \frac{B_{t-1}}{P_{t}} .
$$

We should remark that, in this model, the demand for short term debt is a function of 
the nominal interest rate, the current output and the demand conditions in the goods market, as clarified by equation (16). Therefore, the type of debt that constrains the government's behavior is the long term debt. The parameter $\psi_{1}$ reflects the solvency condition of the government, and measures the reaction of fiscal policy to the level of long term debt. A fiscal policy that follows rule (30) is entirely defined according to the size of parameter $\psi_{1}$. Thus, according to Leeper (1991),

Definition 1 Fiscal policy is passive if

$$
\left|\beta^{-1}-\psi_{1}\right|<1
$$

and active otherwise.

Condition (31) defines the size of parameter $\psi_{1}$ to be satisfied in order to get a non-explosive path for debt.

Finally, public expenditure $G_{t}$ is exogenous according to

$$
\log G_{t}=\left(1-\rho_{G}\right) G+\rho_{G} \log G_{t-1}+\varepsilon_{G t}
$$

where $\varepsilon_{G t}$ is an i.i.d. random variable distributed normally with zero mean and variance $\sigma_{g}^{2}$.

\subsection{Monetary policy}

The central bank follows a variant of the simple policy rule proposed by Taylor (1993)

$$
i_{t}=i\left(\frac{\pi_{t}}{\pi}\right)^{\phi_{\pi}}\left(\frac{x_{t}}{x}\right)^{\phi_{x}}
$$

The nominal short-term interest rate $i_{t}$ reacts to inflation $\pi_{t}$ and output gap $x_{t}$ with intensities captured by the coefficients $\phi_{\pi}$ and $\phi_{x}$.

\section{Aggregate equilibrium}

An important aspect for a full characterization of the equilibrium is represented by the role of intertemporal discount factor of both firms and consumers. We assume that each agent has access to a set of complete market for contingent claims. The direct implication of this assumption is that the discount factor of households should equal that of firms, as stated by

$$
\frac{\rho_{t+1}}{\rho_{t}}=\frac{\beta \lambda_{t+1}}{\lambda_{t}}
$$


For the intuition behind condition (34) it is enough to imagine the presence of a representative agent who can freely exchange shares of each firm, without paying any transaction cost.

Next, in order to reduce the model we can concentrate around a symmetric equilibrium where the choices made by each agent $j$ are the same across all agents. This way, we can assume that $X_{i t}(j)=X_{t}$ for all $i$ and $j$. Therefore, by combining the First Order conditions on consumption and labor (9)-(10) together with (15), the labor supply curve is

$$
\frac{(1-\gamma)}{\gamma} \frac{C_{t}}{1-L_{t}}=\frac{W_{t}}{1+\frac{\chi}{2} F_{t}^{2}+\chi F_{t}}
$$

From equation (35) it is not difficult to show that labor supply function is increasing with respect to real wage, but decreasing with respect to consumption. After making use of the equation of the government budget constraint, we can write the aggregate resource constraint

$$
C_{t}\left(1+\frac{\chi}{2} F_{t}^{2}\right)=Y_{t}\left[1-\frac{\phi_{p}}{2}\left(\pi_{t}-\pi\right)^{2}\right]-G_{t}
$$

From (36) we observe that the amount of income available for consumption is obtained net of resources employed for making transactions and public expenditure. An important feature considered in the present model derives from the specific functional form assumed for the transaction costs function (15). In fact, from Euler equation (12) we find that

$$
\left(1+i_{t}\right) \beta \frac{\lambda_{t+1}}{\pi_{t}}=\lambda_{t}\left(1-\chi F_{t}^{3}\right)
$$

where, from (9) $\lambda_{t}$ is defined according to

$$
\lambda_{t}=\frac{C_{t}^{\gamma\left(1-\frac{1}{\sigma}\right)-1}\left(1-L_{t}\right)^{(1-\gamma)\left(1-\frac{1}{\sigma}\right)}}{\left(1+\frac{\chi}{2} F_{t}^{2}+\chi F_{t}\right) P_{t}}
$$

Therefore, since $\lambda_{t}$ is a monotone decreasing function of $F_{t}$, there are at least two positive steady state satisfying (37). The first is such that $\lambda=0$ with $F=\infty$. The second takes the form

$$
\frac{(1+i) \beta}{\pi}=1-\chi F^{3}
$$

There might be no solution to equation (39). This occurs if $(1+i) \beta>\pi$. If we assume that $(1+i) \beta<\pi$, we immediately get that equation (37) becomes a difference equation, with a converging solution. 


\subsection{A constraint on bond velocity}

Here we briefly sketch an argument in order to exclude that the 'bond velocity' term $F$ grows arbitrarily without bound with positive probability. Let $F$ be the unique solution to (39). If we have an off-equilibrium path value for $F_{t}$ such that $F_{t}>F$ or $\beta<\pi$, then from equation (37) we find that

$$
E_{t}\left[\lambda_{t+1}\right]<\Phi \lambda_{t}
$$

for a given $\Phi$, such that $0<\Phi<1$. Therefore, given the information available at time $t$ condensed by the information set $I_{t}$, the probability that $\lambda_{t+1}$ is lower than $\lambda_{t}$ is positive, given equation (40), i.e.

$$
P\left[\lambda_{t+1}<\Phi \lambda_{t} \mid I_{t}\right]>0
$$

Applying (41) recursively, we get

$$
P\left[\lambda_{t+s}<\Phi^{s} \lambda_{t} \mid I_{t}\right]>0
$$

Equation (41) together with the transaction technology function puts an upper bound on $F$. This allows to exclude any path for $\lambda_{t}$ that require $F$ growing with positive probability. To sum up, if $F_{t}>F$ for all $t$, then $\lambda_{t}$ must have a positive probability of growing arbitrarily close to zero as $t \rightarrow \infty$. However, this would imply a non-zero probability of arbitrarily large values of $F_{t}$. Therefore, $F_{t}>F$ is impossible on an equilibrium path. By a similar argument, if $\beta>\pi$, we have a positive probability of getting an arbitrarily large large value of $F$, which is inconsistent with an equilibrium positive level of short-term debt.

From the same argument, if $F_{t}<F$ and $\beta>\pi$ for some $t$, we have that there is a non-zero probability that $\lambda_{t+s}$ becomes arbitrarily large, so $F_{t}$ assumes values arbitrarily close to zero, as $t \rightarrow \infty$. Again, this is inconsistent with the equilibrium path, because it violates the transversality condition. Again, we must conclude that the value for $F$ that solves (39) is an equilibrium value.

\section{Loglinearized model}

The next step consists in reducing the model into a three equations system an intertemporal version of the IS equation, the aggregate supply equation (AS, henceforth) and the government budget constraint. In order to derive the reduced form system, we take a log-linear approximation around the steady state. In what follows, each variable $X_{t}$ is approximated around the steady state by using the formula. Via 
recursive substitution, we obtain log-linearized version of the resource constraint

$$
\widetilde{C}_{t}=\frac{\widetilde{Y}_{t}}{S_{c}\left(1+\chi F^{2}\right)}-\frac{g}{S_{c}\left(1+\chi F^{2}\right)} \widetilde{G}_{t}-\frac{2 S_{c} \chi F^{3} b_{\zeta}}{S_{c}\left(1+\chi F^{2}\right)} \widetilde{\zeta}_{t}+\frac{2 S_{c} \chi F^{2} b_{i}}{S_{c}\left(1+\chi F^{2}\right)} \widetilde{i}_{t}
$$

where the coefficients $b_{\zeta}, b_{i}$, are reported in Appendix A.

We then log-linearize the first-order conditions (9) and (10), eliminate $\widetilde{C}_{t}$ from both equation, by making use of (43). Next, we can eliminate $\widetilde{L}_{t}$ from the loglinearized version of the production function to obtain

$$
\widetilde{\lambda}_{t}=\eta_{y} \widetilde{Y}_{t}-\eta_{g} \widetilde{G}_{t}+\eta_{a} \widetilde{A}_{t}+\eta_{\zeta} \widetilde{\zeta}_{t}+\eta_{i} \widetilde{i}_{t}
$$

with the coefficients $\eta_{y}, \eta_{g}, \eta_{a}, \eta_{\zeta}, \eta_{i}$.

Finally, from equation (equation (11)), we obtain the intertemporal IS equation

$$
\widetilde{Y}_{t+1}-\lambda_{g} \widetilde{G}_{t+1}+\lambda_{a} \widetilde{A}_{t+1}+\lambda_{\zeta} \widetilde{\zeta}_{t+1}+\lambda_{i} \widetilde{i}_{t+1}-\lambda_{\pi} \widetilde{\pi}_{t+1}=\widetilde{Y}_{t}-\lambda_{g} \widetilde{G}_{t}+\lambda_{a} \widetilde{A}_{t}+\gamma_{\zeta} \widetilde{\zeta}_{t}+\gamma_{i} \widetilde{i}_{t}
$$

where the coefficients are defined as $\lambda_{g}=\eta_{g} / \eta_{y}, \lambda_{a}=\eta_{a} / \eta_{y}, \lambda_{\zeta}=\eta_{\zeta} / \eta_{y}, \lambda_{i}=\eta_{i} / \eta_{y}$, $\gamma_{\zeta}=\left(\eta_{\zeta}-\alpha_{i}\right) \eta_{y}^{-1}, \lambda_{\pi}=\eta_{y}^{-1}$. Equation (45) is the intertemporal IS equation discussed by McCallum and Nelson (1999) and Rotemberg and Woodford (1999). The new feature of equation (45) is the presence of the nominal interest rate dated at time $t+1, \widetilde{i}_{t+1}$, together with the interest rate dated at time $t, \widetilde{i}_{t}$. The presence of $\widetilde{i}_{t+1}$ is due to the characteristics of the transaction technologies $F$ and $B$ considered in the model.

The derivation of the aggregate supply equation starts from the log-linearization of the elasticity $\psi_{t}$, which takes the form

$$
\widetilde{\psi}_{t}=\phi_{p} \widetilde{\pi}_{t}-\beta \phi_{p} \widetilde{\pi}_{t+1}
$$

To simplify the algebra, we assume that the steady state of the inflation rate has been set equal to 1 . From the first order condition with respect to $L$ we have that

$$
\widetilde{W}_{t}=\widetilde{Y}_{t}-\widetilde{L}_{t}+(\theta-1)^{-1} \phi_{p} \widetilde{\pi}_{t}-(\theta-1)^{-1} \beta \phi_{p} \widetilde{\pi}_{t+1}
$$

Moreover, from the production function $\widetilde{L}_{t}=\alpha^{-1}\left(\widetilde{Y}_{t}-\widetilde{A}_{t}\right)$. To get an useful expression of the AS equation, we can substitute out into the log-linearized version of (10), the equation (47) for $\widetilde{W}_{t}$, equation (44) for $\widetilde{\lambda}_{t}$, equation (43) for $\widetilde{C}_{t}$, and $\widetilde{L}_{t}$. After rearrangement, we find

$$
\beta E_{t} \widetilde{\pi}_{t+1}=\widetilde{\pi}_{t}-\mu_{y} \widetilde{Y}_{t}+\mu_{g} \widetilde{G}_{t}+\mu_{\zeta} \widetilde{\zeta}_{t}+\mu_{A} \widetilde{A}_{t}+\mu_{i} \widetilde{i}_{t}
$$


where the coefficients $\mu_{y}, \mu_{g}, \mu_{A}, \mu_{\zeta}, \mu_{i}$ are reported in Appendix A.

The model is expressed as a function of the output gap $X_{t}$ defined (in log-linear terms) as $\widetilde{X}_{t}=\widetilde{Y}_{t}-\widetilde{Y}_{t}^{p}$, where $\widetilde{Y}_{t}^{p}$ is the level of potential output (or full employment level of output) defined as

$$
\widetilde{Y}_{t}^{p}=\frac{\mu_{g}}{\mu_{y}} \widetilde{G}_{t}+\frac{\mu_{\zeta}}{\mu_{y}} \widetilde{\zeta}_{t}+\frac{\mu_{A}}{\mu_{y}} \widetilde{A}_{t}
$$

Therefore, the aggregate supply equation can be written as

$$
\beta E_{t} \widetilde{\pi}_{t+1}=\widetilde{\pi}_{t}-\mu_{y} \widetilde{X}_{t}+\mu_{i} \widetilde{i}_{t}
$$

By the same argument, we can rewrite the IS equation by using the definition of output gap (49), to get

$$
\widetilde{X}_{t+1}+\lambda_{i} \widetilde{i}_{t+1}-\lambda_{\pi} \widetilde{\pi}_{t+1}=\widetilde{X}_{t}+\gamma_{i} \widetilde{i}_{t}+R_{t}^{n}
$$

where $R_{t}^{n}$ indicates a composite term of disturbances

$$
R_{t}^{n}=\left[\delta_{g}\left(\widetilde{G}_{t}-\widetilde{G}_{t+1}\right)+\delta_{a}\left(\widetilde{A}_{t}-\widetilde{A}_{t+1}\right)+\delta_{\zeta 2} \widetilde{\zeta}_{t}-\delta_{\zeta} \widetilde{\zeta}_{t+1}\right]
$$

The term $R_{t}^{n}$ defined in (52) indicates the natural rate of interest from the value of the real interest rate consistent with a full employment equilibrium and a zero inflation rate.

We should stress that, differently what is proposed in the literature, the aggregate supply curve (50) includes the nominal interest rate $\widetilde{i}_{t}$. The presence of $\widetilde{i}_{t}$ is due to the relationship existing between the level of real debt $b_{t}$ and the nominal interest rate that originates from the direct dependence of the Lagrange multiplier from nominal rate $i_{t}$.

Since the present model considers the issue of price level determination on the basis of fiscal solvency, a key equation of the above system is the government budget constraint. In order to make the system entirely defined by four variables (inflation rate, output gap, interest rate and short-term real debt), we can now derive the semi-reduced form of the government budget constraint

$$
\gamma_{y} \widetilde{X}_{t}+\widetilde{b}_{t}^{*}+\gamma_{\pi} \widetilde{\pi}_{t}+\gamma_{i} \widetilde{i}_{t}=\left(\beta^{-1}-\psi_{1}\right) \widetilde{b}_{t-1}^{*}+\theta_{i} \widetilde{i}_{t-1}+\theta_{y} \widetilde{X}_{t-1}+R_{2 t}
$$


where $R_{2 t}$ is defined as

$$
\begin{aligned}
R_{2 t}=\left(\theta_{g}+\theta_{y} \frac{\mu_{g}}{\mu_{x}}\right) \widetilde{G}_{t-1}-\gamma_{g} \widetilde{G}_{t} & +\theta_{y} \frac{\mu_{a}}{\mu_{x}} \widetilde{A}_{t-1} \\
& \quad-\gamma_{y} \frac{\mu_{a}}{\mu_{x}} \widetilde{A}_{t}++\left(\theta_{\zeta}+\theta_{y} \frac{\mu_{\zeta}}{\mu_{x}}\right) \widetilde{\zeta}_{t-1}-\gamma_{\zeta} \widetilde{\zeta}_{t}
\end{aligned}
$$

with all the coefficients reported in Appendix A.

Summing up, the system is made of three equations that are function of the nominal interest rate. After substituting out the reaction function of the central bank, we get a model in three equations and three unknowns $\pi_{t}, X_{t}$ and $b_{t}{ }^{2}$

\section{Calibration}

In order to characterize the bounds for the monetary policy parameters, we provide a set of values for the 'core' parameters of the model, obtained according to a calibration procedure on the basis of quarterly observations drawn for the US economy over the sample 1959:1-2007:4. The calibrated values for the non-policy parameters are reported in Table 1.

The level of the discount factor delivers a value for the real interest rate (in gross terms) equal to 1.003 per quarter, which is consistent with the empirical observations on US economy. The nominal interest rate has been set equal to 1.6 per cent per quarter, as recovered from sample observations. In order to simplify algebra, the long run inflation rate (in gross terms) has been set equal to 1 .

The share of consumption in GDP at the steady state is assumed to be 0.57 . The value of $\chi$ has been set to match a level of transaction costs equal to 2 per cent per year. The elasticity of substitution $\gamma$ between consumption and leisure is set to 0.76 , in order to match the long-run ratio of market to non-market activities $\frac{L}{1-L}=0.2243$ suggested by Christiano (1991).

The elasticity of substitution between different goods variety $\theta$ has been set to be equal to 10, implying a mark up equal to 1.1, as it is customary in the current literature. The elasticity of intertemporal substitution (the inverse of risk aversion coefficient) $\sigma$ has been set to be 0.1 . The estimated values tend is close to 0.08 . The parameter $\alpha$ indicates the share of consumption in the production process as proposed by the large part of the real business cycle literature. Finally the parameter $\phi_{0}$ in the fiscal policy reaction function has been set to match the steady state ratio of debt to GDP equal to 0.44 . The parameter $\psi_{1}$ describing the maginal the reaction of taxes to real debt is set to a benchmark value of 0.05 , which lies within the range

\footnotetext{
${ }^{2}$ In what follows, we drop the tilde sign from each variable. Hence all the variables are expressed in log-linear deviations from the steady state.
} 
established by inequality (31). We calibrate the parameter $A$ to match the postWorld War II level of output of the U.S. economy.

The parameters of the stochastic side of the model are reported in Table 2. The values for $\rho_{A}$ and $\sigma_{A}^{2}$ are from $\operatorname{Kim}(2000)$, while the values for $\rho_{G}$ and $\sigma_{G}^{2}$ are from Schmitt - Grohé and Uribe (2002). For what concerns the value of the parameters of the equation (18). The steady state value for $\zeta_{t}$ has been obtained from the average spread existing between a one-month Treasury Bill and the 10-year government bonds for the US economy. From the dataset, we have $\zeta_{t}=0.24$ on average over the all sample. We estimate equation (18) and obtain $\rho_{\zeta}$ and $\sigma_{\zeta}^{2}=.00196 .^{3}$

\section{Determinacy of a REE}

In this section we analyze the determinacy conditions for two specifications of the central bank's reaction function, namely the cases of pure inflation targeting and standard Taylor rule.

\subsection{Targeting current inflation}

Here we consider the loglinearized interest rate rule

$$
i_{t}=\phi_{\pi} \pi_{t}
$$

According to (55), monetary authority targets only the current inflation rate. A rule like (55) is a very simple representation of the pure inflation target regime, and it represents a good approximation of the European Central Bank operating procedures. After plugging rule (55) in the system (50)-(53) and rearranging, we get a three-equation system in $\pi_{t}, X_{t}, b_{t}$ that can be represented in matrix framework as follows ${ }^{4}$

$$
A Z_{t+1}=B Z_{t}
$$

where vector $Z_{t}$ and matrices $A$ and $B$ are defined as

$$
Z_{t}=\left[\begin{array}{c}
\pi_{t} \\
X_{t} \\
b_{t}^{*}
\end{array}\right]
$$

\footnotetext{
${ }^{3}$ Equation (18) has been estimated by instrumental variables, with four lags of $\zeta_{t}$ as instruments.

${ }^{4}$ Note that from equation (51) and (53) we left out the term $R_{t}^{n}$ and $R_{2 t}$ since they do not affect the dynamic of the system at all.
} 


$$
\begin{aligned}
A & =\left[\begin{array}{ccc}
\beta & 0 & 0 \\
\lambda_{i} \phi_{\pi}-\lambda_{\pi} & 1 & 0 \\
a_{1} & \gamma_{y} & 1
\end{array}\right] \\
B & =\left[\begin{array}{ccc}
1+\mu_{i} \phi_{\pi} & -\mu_{x} & 0 \\
\gamma_{i} \phi_{\pi} & 1 & 0 \\
b_{3} & b_{2} & \beta^{-1}-\psi_{1}
\end{array}\right]
\end{aligned}
$$

with $a_{1} \equiv \gamma_{i} \phi_{\pi}+\gamma_{\pi}, b_{2} \equiv \gamma_{i} \phi_{x}+\gamma_{y}, b_{3} \equiv \theta_{i} \phi_{x}+\theta_{y}$. By inverting matrix A and multiplying matrix $B$ by $A^{-1}$, the system can be cast in the form

$$
Z_{t+1}=\Gamma Z_{t}
$$

with $\Gamma$

$$
\Gamma=\left[\begin{array}{ccc}
\beta^{-1}\left(1+\mu_{i} \phi_{\pi}\right) & -\beta \mu_{x} & 0 \\
\gamma_{21} & \gamma_{22} & 0 \\
\gamma_{31} & \gamma_{32} & \beta^{-1}-\psi_{1}
\end{array}\right]
$$

where the terms $\gamma_{21}, \gamma_{22}, \gamma_{31}, \gamma_{32}$ in (61) are defined as

$$
\begin{aligned}
\gamma_{21} & =\beta^{-1}\left(1+\mu_{i} \phi_{\pi}\right)\left(\lambda_{\pi}-\lambda_{i} \phi_{\pi}\right)+\gamma_{i} \phi_{\pi} \\
\gamma_{22} & =1-\beta^{-1}\left(\lambda_{\pi}-\lambda_{i} \phi_{\pi}\right) \\
\gamma_{31} & =\left[\gamma_{y}\left(\lambda_{i} \phi_{\pi}-\lambda_{\pi}\right)-\gamma_{i}\right]\left(1+\mu_{i} \phi_{\pi}\right)+b_{3}-a_{1} \gamma_{i} \phi_{\pi}
\end{aligned}
$$

From (61) we immediately observe that the structure of the system is blocktriangular. Therefore, to study determinacy we can restrict our attention to the $2 \times 2$ submatrix $\Delta^{2 \times 2}$

$$
\Delta=\left[\begin{array}{cc}
\beta^{-1}\left(1+\mu_{i} \phi_{\pi}\right) & -\beta \mu_{x} \\
\gamma_{21} & \gamma_{22}
\end{array}\right]
$$

To get determinacy for the full system we require that two eigenvalues of the system be outside the unit circle and one inside, since public debt is a predetermined variable. This allows a richer configuration of determinacy conditions rather than in cases without an explicit role of the government budget constraint.

The conditions for determinacy are

Proposition 2 Let $\phi_{\pi} \geqslant 0$. Under contemporaneous pure inflation targeting rule, necessary and sufficient conditions for a rational expectations equilibrium to be determinate are that (a) either

$$
\phi_{\pi}>\phi_{\pi 1} \text { and } \beta^{-1}-1<\psi_{1}<\beta^{-1}+1
$$


(b) or

$$
\phi_{\pi}<\phi_{\pi 1} \text { and } \psi_{1}<\beta^{-1}-1 \quad \psi_{1}>\beta^{-1}+1
$$

with $\phi_{\pi 1}=\frac{\mu_{x} \lambda_{\pi}-2(1+\beta)}{\left[2 \mu_{i}+\mu_{x}\left(\gamma_{i}+\lambda_{i}\right)\right]}$.

\section{Proof 1 See Appendix $C$.}

According to Leeper's (1991) terminology, condition (63) identifies a combination of active monetary and passive fiscal policy, while condition (64) identifies a combination of passive monetary and active fiscal. In the Leeper's sense, an active monetary policy is defined when monetary authority sets nominal interest rate (or money supply) in order to keep under control the inflation rate. A passive monetary policy is when, instead, the interest rate (or money supply) are left free to adjust. In the recent monetary policy literature, condition (64) is identified with a combination of active fiscal policy and passive monetary policy. In particular, if $\phi_{\pi}>\phi_{\pi 1}$ and $\psi_{1}<\beta^{-1}-1, \psi_{1}>\beta^{-1}+1$, we have three roots inside the unit circle. In this case, we have three converging roots and a continuum of solution. In this case the price level is converging, but indeterminate.

\subsection{Taylor rule}

After log-linearizing the Taylor rule around the steady state, we obtain the reaction function

$$
i_{t}=\phi_{\pi} \pi_{t}+\phi_{x} X_{t}
$$

Rule (65) is the standard Taylor rule proposed by Taylor (1993, 1999). According to (65), nominal interest rate reacts contemporaneously with respect to current inflation and output gap. After inserting (65) into (50) and (51) and rearranging we still get a system written in format (60), where now matrix $\Gamma$ is defined according to

$$
\begin{aligned}
\Gamma & =\left[\begin{array}{cc}
\Gamma_{11}^{2 \times 2} & \Gamma_{12}^{2 \times 1} \\
\Gamma_{21}^{1 \times 2} & \Gamma_{22}^{1 \times 1}
\end{array}\right] \\
& =\left[\begin{array}{ccc}
\beta^{-1}\left(1+\mu_{i} \phi_{\pi}\right) & \beta\left(\mu_{i} \phi_{x}-\mu_{x}\right) & 0 \\
\alpha_{21} & \alpha_{22} & 0 \\
\alpha_{31} & \alpha_{32} & \beta^{-1}-\psi_{1}
\end{array}\right]
\end{aligned}
$$

with

$$
\alpha_{21}=\frac{\left(\lambda_{\pi}-\lambda_{i} \phi_{\pi}\right)\left(1+\mu_{i} \phi_{\pi}\right)}{\beta\left(1+\lambda_{i} \phi_{x}\right)}+\frac{\gamma_{i} \phi_{\pi}}{1+\lambda_{i} \phi_{x}}
$$




$$
\begin{aligned}
& \alpha_{22}=\frac{\left(\lambda_{\pi}-\lambda_{i} \phi_{\pi}\right)\left(\mu_{i} \phi_{x}-\mu_{x}\right)+\beta\left(1+\gamma_{i} \phi_{\pi}\right)}{\beta\left(1+\lambda_{i} \phi_{x}\right)} \\
& b_{31}=\frac{\left(\gamma_{y}+\gamma_{i} \phi_{x}\right)\left(\lambda_{i} \phi_{\pi}-\lambda_{\pi}\right)}{\beta\left(1+\lambda_{i} \phi_{x}\right)}-\frac{\left(\gamma_{\pi}+\gamma_{i} \phi_{\pi}\right)}{\beta} \\
& b_{32}=\frac{\gamma_{y}+\gamma_{i} \phi_{x}}{1+\lambda_{i} \phi_{x}} \\
& \alpha_{31}=b_{31}\left(1+\mu_{i} \phi_{\pi}\right)+b_{32} \gamma_{i} \phi_{\pi}+\theta_{i} \phi_{\pi} \\
& \alpha_{32}=b_{31}\left(\mu_{i} \phi_{x}-\mu_{x}\right)+b_{32}\left(1+\gamma_{i} \phi_{\pi}\right)+\theta_{y}+\theta_{i} \phi_{x} .
\end{aligned}
$$

Proposition 3 Given $\phi_{\pi} \geqslant 0, \phi_{x} \geqslant 0$. For a model with contemporaneous inflation and output targeting interest rules, necessary and sufficient conditions for a REE to be determinate are that

(i) either

$$
\underline{\phi}_{\pi}<\phi_{\pi}<\bar{\phi}_{\pi} \text { and } \beta^{-1}-1<\psi_{1}<\beta^{-1}+1
$$

(ii) or

$$
\phi_{\pi}<\underline{\phi}_{\pi} \text { and } \quad \psi_{1}<\beta^{-1}-1 ; \quad \psi_{1}>\beta^{-1}+1
$$

where

$$
\begin{aligned}
\bar{\phi}_{\pi} & =\frac{1-\beta+\left(\lambda_{\pi}-1\right) \mu_{x}+\phi_{x}\left(\gamma_{i}-\lambda_{i}-\lambda_{\pi} \mu_{i}-\beta \gamma_{i}\right)}{\lambda_{i} \mu_{i}} \\
\underline{\phi}_{\pi} & =\frac{\mu_{x}\left(1+\lambda_{\pi}\right)-2(1+\beta)-\phi_{x}\left(\lambda_{i}+\lambda_{\pi} \mu_{i}+\gamma_{i}(1+\beta)\right)}{2 \mu_{i}+\lambda_{i} \mu_{x}}
\end{aligned}
$$

Proof 2 See Appendix D.

Even in this case we can confirm the same results discussed in the previous case with a pure inflation targeting rule. From condition (74) we have that a combination of active-monetary with passive -fiscal delivers a fully determinate equilibrium. Since matrix $\Gamma$ is once again lower triangular to guarantee a determinate REE we need to have two roots outside the unit circle. Because of the format of matrix $\Gamma$, the requirement of active monetary policy can be violated, but the equilibrium is still determinate if fiscal policy is properly set to keep the price level determinate.

The nature of the bounds determined by (74) and (75) is more complex than in the pure inflation targeting case. This is because the upper and lower limits for the inflation targeting parameter $\phi_{\pi}$ are now a function of the output gap targeting parameter $\phi_{x}$. According to the benchmark parameter values highlighted in Table 1 , we get that the relationship existing between $\phi_{\pi}$ and $\phi_{x}$ is increasing, as represented in Figure 1. This figure has been obtained by varying $\phi_{x} \in[0,2]$. In order 
to keep the equilibrium determinate, under a standard Taylor rule, the inflation targeting parameter must increase as $\phi_{x}$ raises. Thus, determinacy is reached only for combination of parameters lying on the line or on above the line that separates the two region where we get either determinacy or indeterminacy. The intuition behind this result is related to the logic underlying the Taylor principle. The inflation targeting parameter should be set to be bigger than one, and the relative magnitude between $\phi_{\pi}$ and $\phi_{x}$ must be kept constant as $\phi_{x}$ raises. Of course, all these considerations hold if a monetary policy rule is associated with a fiscal policy rule that sets the primary surplus as a function of real debt under the conditions established by (74). When, instead the conditions are given by (75), the regions with determinacy/indeterminacy is reversed, with respect to what has been represented in Figure 1. In this last case, monetary policy need not be active provided that fiscal policy is set according to (75).

\section{Conclusion}

In this paper we discuss the determinacy of the price level in a cashless environment. We present a model with two types of government bonds, a short-term bond that is used for the purchase of consumption goods, and a long-term bond that is used as a store of value. If fiscal policy is designed according to the prescriptions of fiscal theory of the price level of Leeper (1991), determinacy is achieved by following the Taylor principle. In order to pin down the price level, fiscal policy should be passive in the sense of Leeper (1991). These findings stand in stark contrast with those of Canzoneri and Diba (2004) and Canzoneri et al. (2006), who use a model with liquidity services from money and bonds to suggest that the Taylor principle is not a panacea for determinacy.

A number of additional questions of interest are raised in our paper. Our results suggest that the introduction of a sophisticated demand for money affects the determinacy properties of simple rules for monetary policy, rather than the macroeconomic role per se played by government bonds. It this sense it would be important to understand whether different modelling assumptions about money matter for the determinacy properties. Finally, the introduction of distortionary taxation adds a new transmission channel that can change the results greatly. 


\section{A Coefficients of the reduced-form model}

$$
\begin{aligned}
b_{\zeta} & =\frac{\zeta i}{3 \chi F^{3}(1+\zeta i)} \\
b_{i} & =\frac{i(1-\zeta)+\zeta i^{2}}{3 \chi F^{3}(1+\zeta i)^{2}}
\end{aligned}
$$

Coefficients of equation (44)

$$
\begin{aligned}
\eta_{y} & =\frac{\gamma\left(1-\frac{1}{\sigma}\right)-1}{S_{c}\left(1+\chi F^{2}\right)}-\frac{(1-\gamma)\left(1-\frac{1}{\sigma}\right) L}{\alpha(1-L)} ; \quad \eta_{a}=\frac{(1-\gamma)\left(1-\frac{1}{\sigma}\right) L}{\alpha(1-L)} ; \\
\eta_{\zeta} & =\frac{\chi F(1+F) b_{\zeta}}{1+\frac{\chi}{2} F^{2}+\chi F}-\frac{\left[\gamma\left(1-\frac{1}{\sigma}\right)-1\right] 2 S_{c} \chi F^{2} b_{\zeta}}{S_{c}\left(1+\chi F^{2}\right)} ; \\
\eta_{g} & =\frac{\left[\gamma\left(1-\frac{1}{\sigma}\right)-1\right] g}{S_{c}\left(1+\chi F^{2}\right)} ; \quad \eta_{i}=\frac{\left[\gamma\left(1-\frac{1}{\sigma}\right)-1\right] 2 S_{c} \chi F^{2} b_{i}}{S_{c}\left(1+\chi F^{2}\right)}-\frac{\chi F(1+F)}{1+\frac{\chi}{2} F^{2}+\chi F}
\end{aligned}
$$

Coefficients of equation (48):

$$
\begin{aligned}
& \mu_{y}=\frac{(\theta-1)}{\phi_{p}}\left\{\frac{\gamma\left(1-\frac{1}{\sigma}\right)}{S_{c}\left(1+\chi F^{2}\right)}-\frac{\left[(1-\gamma)\left(1-\frac{1}{\sigma}\right)-1\right] \frac{L}{(1-L)}-1}{\alpha}-\eta_{y}-1\right\} \\
& \mu_{g}=\frac{(\theta-1)}{\phi_{p}}\left[\frac{\gamma\left(1-\frac{1}{\sigma}\right) g}{S_{c}\left(1+\chi F^{2}\right)}-\eta_{g}\right] \\
& \mu_{i}=\frac{(\theta-1)}{\phi_{p}}\left[\eta_{i}-\frac{2 S_{c} \chi F^{2} b_{i} \gamma}{S_{c}\left(1+\chi F^{2}\right)}\left(1-\frac{1}{\sigma}\right)\right] \\
& \mu_{\zeta}=\frac{(\theta-1)}{\phi_{p}}\left[\eta_{\varsigma}+\frac{2 S_{c} \chi F^{2} b_{\zeta} \gamma}{S_{c}\left(1+\chi F^{2}\right)}\left(1-\frac{1}{\sigma}\right)\right] \\
& \mu_{A}=\frac{(\theta-1)}{\phi_{p}}\left\{\eta_{A}-\frac{\left[(1-\gamma)\left(1-\frac{1}{\sigma}\right)-1\right] \frac{L}{(1-L)}-1}{\alpha}\right\}
\end{aligned}
$$


The coefficients of equation (53) are defined as

$$
\begin{aligned}
\gamma_{y} & =\frac{b}{b^{*}} \frac{1}{S_{c}\left(1+\chi F^{2}\right)} ; \quad \gamma_{\zeta}=\frac{b}{b^{*}} \frac{2 \chi F^{3} b_{\zeta}}{\left(1+\chi F^{2}\right)} \quad \alpha_{i}=\frac{2 \chi F^{2} b_{i}}{\left(1+\chi F^{2}\right)} \\
\gamma_{i} & =\frac{b}{b^{*}} \alpha_{i} ; \quad \gamma_{g}=\frac{1}{b^{*}}\left(b \frac{g}{S_{c}\left(1+\chi F^{2}\right)}+G\right) \\
\theta_{i} & =i^{*}+\frac{i b}{b^{*}}+(1+i) \frac{b \alpha_{i}}{b^{*}}+\frac{b}{b^{*}} \frac{2 \chi F^{2} b_{i} \psi_{2}}{\left(1+\chi F^{2}\right)}+\frac{\psi_{2} b b_{i}}{b^{*}} \\
\gamma_{\pi} & =i^{*}+\frac{i b}{b^{*}}-\psi_{1}-\psi_{2} \frac{b}{b^{*}} \\
\theta_{\zeta} & =i^{*}-(1+i) \frac{b}{b^{*}}\left[\frac{2 \chi F^{3} b_{\zeta}}{\left(1+\chi F^{2}\right)}\right]+\frac{2 b \chi F^{3} b_{\zeta} \psi_{2}}{b^{*}\left(1+\chi F^{2}\right)}-\frac{\psi_{2} b b_{\zeta}}{b^{*}} \\
\theta_{y} & =\frac{b\left(1+i-\psi_{2}\right)}{b^{*} S_{c}\left(1+\chi F^{2}\right)} ; \quad \theta_{g}=\frac{b\left[\psi_{2}-(1+i) g\right]}{b^{*} S_{c}\left(1+\chi F^{2}\right)}
\end{aligned}
$$

\section{B Schur-Cohn criterion}

The characteristic equation of a $2 \times 2$ matrix $A$ is $x^{2}-\operatorname{tr}(A) x+\operatorname{det}(A)=0$. It is well known that the condition for two roots of the characteristic equation to lie outside the unit circle is (see LaSalle, 1986)

$$
\begin{aligned}
|\operatorname{det}(A)| & >1, \\
|\operatorname{tr}(A)| & <1+\operatorname{det}(A) .
\end{aligned}
$$

In particular, condition (79) can be split up in the two inequalities

$$
\begin{aligned}
& 1+\operatorname{det}(A)+\operatorname{tr}(A)>0 \\
& 1+\operatorname{det}(A)-\operatorname{tr}(A)>0
\end{aligned}
$$

\section{Proof of proposition 2}

The trace and the determinant of submatrix $\Delta$ defined in (62) are

$$
\begin{aligned}
\operatorname{det}(\Delta) & =\frac{1}{\beta}+\frac{\left(\mu_{i}+\mu_{x} \gamma_{i}\right) \phi_{\pi}}{\beta} \\
\operatorname{tr}(\Delta) & =1+\frac{1}{\beta}+\frac{\left(\mu_{i}+\mu_{x} \gamma_{i}\right) \phi_{\pi}}{\beta}-\frac{\mu_{x} \lambda_{\pi}}{\beta}
\end{aligned}
$$

From condition (78) of the Schur-Cohn criterion, it is certainly true that $\operatorname{det}(\Delta)>$ -1 . The condition $\operatorname{det}(\Delta)>1$ implies

$$
\phi_{\pi}>-\frac{(1-\beta)}{\mu_{i}+\mu_{x} \gamma_{i}}
$$


On the other hand, condition (80) directly implies

$$
\phi_{\pi}>\frac{\mu_{x} \lambda_{\pi}-2(1+\beta)}{\left[2 \mu_{i}+\mu_{x}\left(\gamma_{i}+\lambda_{i}\right)\right]}
$$

while (81)

$$
\phi_{\pi}\left(1+\gamma_{i}-\lambda_{i}\right)>0
$$

which is always satisfied since we set $\phi_{\pi}>0$. By using the benchmark parameter values considered in the model, it is immediate to verify that the bound established by (83) is bigger than that specified by (82), under a wide range of the core parameter values. Therefore, condition (83) is both necessary and sufficient to ensure determinacy. For the system condensed in matrix $\Gamma$ we require that the third root be inside the unit circle. This is true if condition (31) is respected, which is equivalent to require that

$$
\beta^{-1}-1<\psi_{1}<\beta^{-1}+1
$$

When both conditions (83) and (85) are satisfied, then all the three roots of the system are inside the unit circle, and the equilibrium is unique.

\section{Proof of proposition 3}

Once again, given the triangular structure of matrix we can concentrate on the eigenvalues of the submatrix $\Gamma_{11}^{2 \times 2}$ and that of $\Gamma_{22}^{1 \times 1}$, in (66)-(67).

The trace and the determinant of submatrix $\Gamma_{11}^{2 \times 2}$ are given, respectively, by

$$
\begin{aligned}
\operatorname{tr}\left(\Gamma_{11}\right) & =\frac{\left(1+\mu_{i} \phi_{\pi}\right)\left(1+\lambda_{i} \phi_{x}\right)+\left(\lambda_{\pi}-\lambda_{i} \phi_{\pi}\right)\left(\mu_{i} \phi_{x}-\mu_{x}\right)+\beta\left(1+\gamma_{i} \phi_{x}\right)}{\beta\left(1+\lambda_{i} \phi_{x}\right)} \\
\operatorname{det}\left(\Gamma_{11}\right) & =\frac{\mu_{i} \phi_{\pi}+1+\gamma_{i} \phi_{x}-\mu_{x}}{\beta\left(1+\lambda_{i} \phi_{x}\right)}
\end{aligned}
$$

The condition $\operatorname{det}(\Delta)>1$ implies

$$
\phi_{\pi}>\frac{\mu_{x}-(1-\beta)-\left(\gamma_{i}-\beta \lambda_{i}\right) \phi_{x}}{\mu_{i}}
$$

On the other hand, from condition (80) we get

$$
\phi_{\pi}>\frac{\mu_{x}\left(1+\lambda_{\pi}\right)-2(1+\beta)-\phi_{x}\left(\lambda_{i}+\lambda_{\pi} \mu_{i}+\gamma_{i}(1+\beta)\right)}{2 \mu_{i}+\lambda_{i} \mu_{x}}
$$


Finally, from (81) we find the constraint

$$
\phi_{\pi}<\frac{1-\beta+\left(\lambda_{\pi}-1\right) \mu_{x}+\phi_{x}\left(\gamma_{i}-\lambda_{i}-\lambda_{\pi} \mu_{i}-\beta \gamma_{i}\right)}{\lambda_{i} \mu_{i}}
$$

According to benchmark parameter values in the calibration section, we immediately get that the only constraints that bind are (87) and (88). In fact, the bound is determined by (86) is equal to 1.6233 , while (87) is equal to 1.74 . Therefore, if (87) is satisfied, so is (86). Finally, the bound in (88) is equal to 153.9. Given the standard values for $\phi_{\pi}$ this is always satisfied ${ }^{5}$.

Conditions (87)-(88) imply that two eigenvalues of matrix $\Gamma$ are outside the unit circle. This is enough to establish the determinacy of the REE induced by rule (65). Thus, the third root given by the determinant of $\Gamma_{22}^{1 \times 1}$ should lie inside the unit circle. This is equivalent to require that condition (31) be verified, or that $\beta^{-1}-1<\psi_{1}<\beta^{-1}+1$, that generates condition (74).

If one of the constraints (87)-(88) is not satisfied, then the submatrix $\Gamma_{11}$ has one root inside and one outside the unit circle. To restore determinacy we need another root outside the unit circle. This can be obtained by setting $\left|\beta^{-1}-\psi_{1}\right|>1$, or $\psi_{1}<\beta^{-1}-1 ; \psi_{1}>\beta^{-1}+1$.

\footnotetext{
${ }^{5}$ Note that for the simulation it has been assumed a value for $\phi_{x}$ equal to 0.5 , as the benchmark values proposed by Taylor (1999).
} 


\section{References}

[1] Canzoneri, M.,B., and B.T. Diba, (2005), "Interest Rate Rules and Price Determinacy: the Role of Transactions Services of Bonds", mimeo, Georgetown University.

[2] Canzoneri, M.,B., R. Cumby, B.T. Diba, and D. Lopez-Salido (2006), "Monetary and Fiscal Policy Coordination when Bonds Provide Transactions Services", mimeo, Georgetown University.

[3] Christiano, L., J., (1991), "Modelling the Liquidity Effect of a Money Shock", Federal Reserve Bank of Minneapolis Quarterly Review, vol. 15, 1.

[4] Clarida, R., Galì, J., and M. Gertler, (2000), "Monetary Policy Rules and Macroeconomic Stability: Evidence and Some Theory", Quarterly Journal of Economics, 115, 147-180.

[5] Goodfriend, M., and R., G., King, (1997), "The New Neoclassical Synthesis and the Role of Monetary Policy", in Ben Bernanke and Julio, J. Rotemberg (eds.), NBERMacroeconomic Annual 1997, the MIT Press, Cambridge, MA.

[6] Kim, J., (2000), "Constructing and Estimating a Realistic Optimizing Model of Monetary Policy", Journal of Monetary Economics, 45, 329-359.

[7] LaSalle, Joseph P. (1986): The Stability and Control of Discrete Processes. Springer Verlag, New-York, Berlin.

[8] Leeper, E.,M., (1991), "Equilibria Under Active and Passive Monetary and Fiscal Policies", Journal of Monetary Economics, 27, 129-47.

[9] McCallum, B., T, and E. Nelson, (1999), "Performance of Operational Policy Rules in an Estimated Semiclassical Structural Model", in J. Taylor (ed.), Monetary Policy Rules, University of Chicago Press, Chicago, IL.

[10] Rotemberg, J., J., (1982), "Sticky Prices in the United States", Journal of Political Economy, 90, 1187-1211.

[11] Rotemberg, J. and M. Woodford (1997): "An Optimization-Based Econometric Framework for the Evaluation of Monetary Policy", in NBER Macroeconomic Annual, edited by Ben Bernanke and Michael Woodford, The MIT Press, Cambridge, MA.

[12] Rotemberg, J. and M. Woodford (1999): "Interest Rate Rules in an Estimated Sticky Price Model", in Monetary Policy Rules, edited by John Taylor, NBERConference Report, University of Chicago Press, Chicago, IL. 
[13] Schmitt-Grohé, S., and M. Uribe, (2001a), "Optimal Fiscal and Monetary Policy Under Sticky Prices", CEPR Working Paper No. 2942, September.

[14] Taylor, J., B., (1993), "Discretion Versus Policy Rules in Practice", CarnegieRochester Conference Series on Public Policy, 39, 195-214.

[15] Woodford, M., (1998), "Doing without Money: Controlling Inflation in a PostMonetary World", Review of Economic Dynamics, 1, 173-219.

[16] Woodford, M., (2002), "Financial Market Efficiency and the Effectiveness of Monetary Policy", Federal Reserve Bank of New York Economic Policy Review, Vol. 8 No. 1, pp. 85-94.

[17] Woodford, M., (2007), "How Important is Money in the Conduct of Monetary Policy?", paper presented at the Conference in Honor of Ernst Baltensperger, University of Bern. 
Table 1: Non-Policy Parameters

\begin{tabular}{lllllllll}
\hline$\beta$ & $\chi$ & $\gamma$ & $\theta$ & $\sigma$ & $\psi_{0}$ & $\psi_{1}$ & $\alpha$ \\
\hline 0.997 & 0.22 & 0.76 & 10 & 0.1 & 8.31 & 0.05 & 0.77 \\
\hline
\end{tabular}


Table 2: Calibration of shocks

\begin{tabular}{lccc}
\hline Parameter & $\rho_{A}$ & $\rho_{\zeta}$ & $\rho_{G}$ \\
\hline Value & 0.98 & 0.74 & 0.64 \\
\hline Parameter & $\sigma_{A}^{2}$ & $\sigma_{\zeta}^{2}$ & $\sigma_{G}^{2}$ \\
\hline Value & 0.0003 & 0.00196 & 0.000126 \\
\hline
\end{tabular}


Figure 1: Determinacy regions with Taylor-type rule

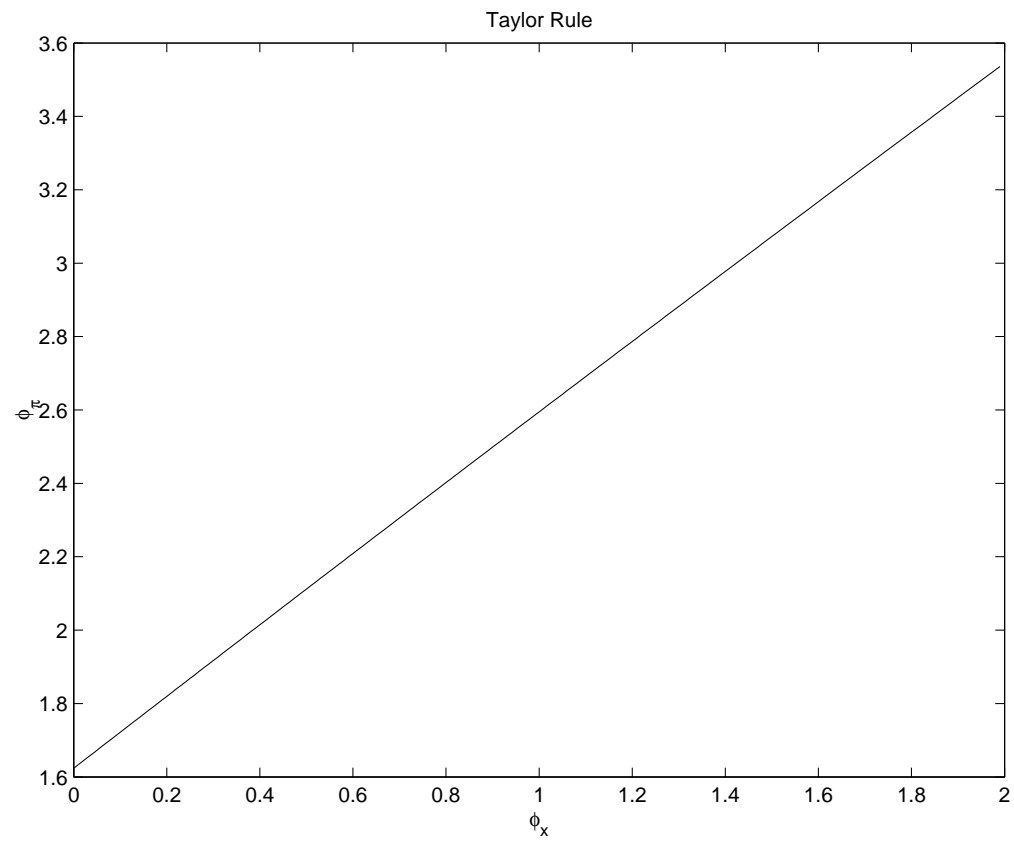

